\title{
Níveis de Sódio em Rações de Poedeiras Comerciais no Segundo Ciclo de Postura1
}

\author{
Eliana Aparecida Rodrigues ${ }^{2}$, Otto Mack Junqueira ${ }^{3}$, Maurício Valério ${ }^{4}$, Marcelo de Oliveira \\ Andreott ${ }^{5}$, Luciana Cardoso Cancherini ${ }^{6}$, Douglas Emygdio de Faria ${ }^{7}$, Rosemeire da Silva Filardi ${ }^{8}$
}

RESUMO - O objetivo deste experimento foi determinar o efeito de diferentes níveis de sódio para poedeiras nos períodos de repouso e segundo ciclo de postura. Foram utilizadas 216 poedeiras da linhagem Hy line W-36, pós-muda forçada, com 60 semanas de idade. O delineamento utilizado foi o inteiramente casualizado em arranjo fatorial 3 x 3 (três níveis de sódio no período de repouso x três níveis de sódio no período de postura), totalizando nove tratamentos com três repetições de oito aves cada. Foram avaliadas, em quatro ciclos de 28 dias, as seguintes variáveis: produção e peso dos ovos, consumo de ração, conversão alimentar, massa de ovos, qualidade do albúmen, percentagem de casca, gravidade específica e espessura das cascas. Não foram encontrados efeitos significativos dos níveis de sódio sobre as variáveis estudadas, entretanto, para a espessura de casca, o menor resultado foi obtido com o nível de $0,15 \%$ de sódio. Os níveis de sódio de $0,15 \%$ no período de repouso e de $0,25 \%$ no período de postura foram suficientes para atender às exigências nutricionais de poedeiras no segundo ciclo de postura.

Palavras-chave: muda forçada, poedeiras comerciais, produção de ovos, qualidade da casca do ovo, sódio

\section{Sodium Levels in Laying Hen Diets in the Second Phase of Egg Production}

\begin{abstract}
One experiment was conducted to evaluate the effects of sodium levels on performance of laying hens in second phase of egg production. Two hundred and sixteen laying hens (Hy-line W-36), after forced molt at 60 weeks of age, were used. The experimental design was a factorial with three sodium levels $(.15, .25$, and $.35 \%)$ during 30 days after fasting period and the same levels on the production period. Four periods of 28 days each were evaluated concerning the following parameters: egg production, feed intake, feed conversion, egg mass, albumen quality, egg percentage, egg specific gravity and shell thickness. No significant effect of the sodium levels on studied parameters was observed. However, the smaller result for shell thickness was found at .15\% sodium level. The level of $.15 \%$ sodium in the fasting period and $.25 \%$ sodium in the production period were enough to met the nutritional requirement of laying hens at the second cycle of egg production.
\end{abstract}

Key Words: forced molt, laying hen, shell quality, sodium levels

\section{Introdução}

A muda forçada atualmente tem sido utilizada como prática comum no Brasil, visto que proporciona economia para o produtor. No entanto, o período pósmuda é conhecido como uma fase em que as aves produzem ovos maiores e com maiores problemas de casca, devido ao rápido aumento do tamanho do ovo e, em consequiência, a espessura da casca e sua porcentagem em relação ao peso do ovo diminuem. Na literatura, existe grande número de estudos relacionados às exigências nutricionais, à qualidade do ovo e ao desempenho produtivo de poedeiras no primeiro ciclo de produção, no entanto, essas informações são escassas para o segundo ciclo de postura. Para essas galinhas que sofreram a muda forçada, normalmente são utilizadas as exigências nutricionais do final do primeiro ciclo de postura, com redução nos teores de nutrientes, o que não é adequado, pois, para ótimo desempenho das aves que sofreram a muda forçada, torna-se necessária uma revisão quanto às suas exigências nutricionais neste período (Christmas \& Harms, 1983).

\footnotetext{
${ }^{1}$ Parte da dissertação da primeira autora. Pesquisa financiada pela FAPESP (Proc.00/12.377-1).

2 Pós-graduanda (Doutorado) em Zootecnia FCAVJ - v.a. Prof. Paulo D.Castellane s/n CEP: 14884-900. E.mail: elianaar@fcav.unesp.br

${ }^{3}$ Professor do departamento de Zootecnia da Faculdade de Ciências Agrárias e Veterinárias de Jaboticabal - UNESP.

E.mail: ottomack@fcav.unesp.br

${ }^{4}$ Aluno da graduação da FCAV/UNESP.

5 Professor da UFMS - Campo Grande - MS. E.mail: andreoti@nin.ufms.br

6 Pós-graduanda (Doutorado) em Zootecnia FCAV/UNESP. E.mail: lucianac @fcav.unesp.br

7 Professor da FZEA/USP - Pirassununga - SP. E.mail: defaria @ usp.br

8 Pós-doutoranda em Zootecnia - FCAV/UNESP. E.mail: rofilardi@ig.com.br
} 
O sódio é essencial para todos os animais, mas, por encontrar-se facilmente disponível na forma de cloreto de sódio e ter baixo custo, recebe pouca atenção dos nutricionistas. O nível de suplementação de cloreto de sódio não é ajustado de acordo com as variações deste elemento na composição dos ingredientes nas dietas. Não apenas o sódio é essencial para todos os animais, mas também o cloro, sendo que, no campo da nutrição, não é comum o estudo destes elementos separadamente, porque sua suplementação, para atender às exigências nutricionais das aves, é feita com uso de sal comum, constituído de aproximadamente $38 \%$ de $\mathrm{Na}$ e $60 \%$ de $\mathrm{Cl}$.

O sódio é um cátion monovalente encontrado nos líquidos extracelulares, principalmente no soro sangüíneo, realizando importantes funções metabólicas. Desse modo, é necessário supri-lo em níveis e balanço adequados para ótimo desempenho das aves. As funções metabólicas do sódio estão intimamente relacionadas com equilíbrio do volume hídrico, $\mathrm{pH}$ e transmissão de impulsos nervosos. McDowell (1992) cita, também como funções do sódio, manutenção de contrações musculares e cardíacas e absorção da água e de algumas vitaminas hidrossolúveis (riboflavina, tiamina e ácido ascórbico). Segundo Scott et al. (1982), os sintomas apresentados pelas aves alimentadas com dietas deficientes em sódio são redução no crescimento, enfraquecimento dos ossos, queratinização da córnea, inatividade gonadal, comprometimento de funções celulares, diminuição na utilização da proteína e energia e redução do volume plasmático. Em poedeiras, a deficiência de sódio resulta em decréscimo ou parada da postura, retardo no crescimento e canibalismo (Kuchinski et al., 1997).

Há muitos anos, a deficiência de sódio já era conhecida por afetar a produção de ovos. Burns et al. (1952) observaram que as poedeiras Leghorn necessitavam de $0,19 \%$ de sal $(0,076 \%$ de $\mathrm{Na})$ em dietas purificadas, sendo que o fator limitante para a produção de ovos, eclodibilidade e manutenção do peso corporal foi o sódio, e não o cloro. Harms (1991), avaliando o efeito da retirada do sal, do sódio e do cloro da dieta de poedeiras com 45 ou 65 semanas de idade, concluiu que o sódio é o principal elemento envolvido na interrupção da postura, quando ausente da dieta.

Quando o cloreto de sódio é retirado da dieta, o sódio mostra-se mais limitante em relação ao cloro, porque seu nível, na maioria dos ingredientes para rações, é mais baixo que o do cloro e, conjuntamente, o sódio e o cloro intervêm no equilíbrio da pressão osmótica, no equilíbrio ácido-básico e na permeabilidade celular (Andriguetto et al., 1986).

Não apenas a deficiência de sódio reflete em prejuízos na produção de poedeiras, mas também seu excesso, uma vez que este macroelemento, assim como o potássio e o cloreto, em doses excessivas, determinam aumento na absorção de água em aves (Hooge, 1999). De acordo com Wideman et al. (1985), aves consumindo rações com altos níveis de sódio apresentam aumento no consumo de água e, conseqüentemente, na umidade das excretas e da excreção urinária de sódio, além de redução nas taxas de filtração glomerular. Esse aumento na umidade das excretas pode representar grande problema, uma vez que essa situação propicia o desenvolvimento de larvas de moscas.

Os níveis de sódio nas rações recomendados para atender às exigências nutricionais de poedeiras comerciais apresentam diferenças entre as diversas fontes de referência. Scott et al. (1982) recomendam $0,12 \%$ de sódio nas dietas para poedeiras em produção, enquanto o NRC (1984 e 1994), Rostagno et al. (1985 e 2000) e Leeson \& Summers (1991) recomendam, respectivamente, 150, 225 e $180 \mathrm{mg}$ de sódio ave/dia. Entretanto, como o sódio atua para manter o equilíbrio eletrolítico dentro dos valores normais, é fundamental considerar o balanço ácido-básico das dietas (Mongin, 1980).

O NRC (1994) recomenda níveis de $0,15 \%$ de sódio e potássio e $0,13 \%$ de cloro para as aves de primeiro ciclo de produção que ingerem $100 \mathrm{~g}$ de ração por dia com $2.900 \mathrm{kcal}$ de EM/kg, mas não faz referências para as aves no período pós-muda. O balanço de $\mathrm{Na}, \mathrm{K}$ e $\mathrm{Cl}$ age diretamente no equilíbrio ácido-base das aves, podendo influenciar seu desempenho, o metabolismo de cálcio, a utilização do fósforo e, ainda, outras funções fisiológicas.

Devido à influência do sódio na produtividade das aves, é necessário fazer uma reavaliação das exigências nutricionais das aves em produção, principalmente durante o segundo ciclo de postura, para que estas possam expressar todo seu potencial produtivo.

O objetivo deste trabalho foi determinar o efeito de níveis de sódio, fornecido sob a forma de cloreto de sódio na ração, sobre o desempenho e a qualidade dos ovos de poedeiras comerciais no segundo ciclo de produção. 


\section{Material e Métodos}

Foram utilizadas 216 poedeiras comerciais da linhagem Hy-line W-36, com 60 semanas de idade, distribuídas em delineamento inteiramente casualizado em esquema fatorial $3 \times 3$ (três níveis de sódio no período de repouso $\mathrm{x}$ três níveis de sódio no período de postura) com três repetições, totalizando 27 parcelas experimentais, sendo cada parcela constituída por oito aves. Após o jejum, durante o período de repouso (30 dias), foram fornecidos três níveis de sódio $(0,15 ; 0,25$ e $0,35 \%)$ e, no posterior período de postura (quatro ciclos de 28 dias), três níveis de sódio $(0,15 ; 0,25$ e $0,35 \%)$. Os níveis de sódio (\%) aplicados nos períodos de repouso e postura são apresentados na Tabela 1.

As dietas experimentais foram isoprotéicas (16\% de proteína bruta) e isocalóricas ( $2800 \mathrm{kcal} \mathrm{de} \mathrm{EM/kg),}$ sendo formuladas segundo as recomendações nutricionais do NRC (1994). A composição percentual das rações, assim como os valores calculados dos respectivos níveis nutricionais, encontra-se na Tabela 2.

As poedeiras foram submetidas a um método de muda forçada, no qual ficaram em jejum e sem o fornecimento de luz artificial até alcançarem perda do peso corporal de, aproximadamente, $25 \%$, o que ocorreu após 13 dias. As aves foram alojadas em galpão convencional de postura com gaiolas de arame galvanizado com uma ave por compartimento. Após o término da muda forçada, voltou-se com a iluminação artificial, com acréscimo de 30 minutos por semana até atingir 17 horas de luz/dia, tornando-se constante até o final do experimento.

Ao final do 30 으 dia pós-jejum, foram avaliadas as características de desempenho e, ao final de cada ciclo de 28 dias, além das características de desempenho, foram avaliadas também as características de qualidade de albúmen e qualidade externa dos ovos. Para avaliar espessura da casca, peso dos ovos e unidade Haugh, foram utilizados quatro ovos por parcela, colhidos nos últimos dois dias do ciclo, com exceção da gravidade específica, em que todos os ovos íntegros produzidos nos dois últimos dias de cada ciclo foram avaliados. Esses ovos foram mergulhados em soluções de $\mathrm{NaCl}$, com densidade entre 1,070 e $1,106 \mathrm{~g} / \mathrm{cm}^{3}$ e gradiente de 0,006 entre elas, sendo essas soluções preparadas conforme recomendações de Moreng \& Avens (1990). A massa dos ovos foi obtida com a multiplicação da produção de ovos (\%) pelo peso médio dos mesmos (g).
Os resultados obtidos no experimento foram submetidos à análise estatística, utilizando-se o programa "ESTAT", desenvolvido pelo Departamento de Ciências Exatas da FCAV - UNESP Jaboticabal. Em caso de significância estatística, as médias foram comparadas pelo teste de Tukey, a 5\% de probabilidade.

\section{Resultados e Discussão}

Os valores médios para produção e peso dos ovos, consumo diário de ração, conversão alimentar, massa dos ovos e unidades Haugh, com os respectivos coeficientes de variação, encontram-se na Tabela 3. O efeito não significativo dos níveis de sódio sobre o peso do ovo era previsto, uma vez que apenas a manipulação de proteína (Keshavarz \& Nakajima, 1995), metionina (Keshavarz, 1995), lisina (Zimmerman, 1997) e ácido linoléico (Scragg et al., 1987) é responsável por essa alteração.

Em relação às características produtivas, não foi verificado efeito significativo $(\mathrm{P}>0,0,5)$ entre os diferentes níveis de sódio. O consumo de ração permaneceu constante com o aumento dos níveis de sódio utilizados; o mesmo efeito foi verificado por Faria et al. (2000), trabalhando com níveis de até $0,24 \%$. No entanto, ao utilizarem $0,43 \%$ de sódio, Junqueira et al. (2000) observaram redução significativa no consumo de ração.

Das características de qualidade da casca (Tabela 4), apenas a sua espessura foi influenciada $(\mathrm{P}<0,05)$ pelos níveis de sódio fornecidos, tendo-se verificado melhora nessa característica, quando o nível de sódio passou de 0,15 para $0,25 \%$. Os níveis de sódio fornecidos no período de repouso não afetaram $(\mathrm{P}>0,05)$ a espessura

Tabela 1 - Níveis de sódio (\%) utilizados nas rações experimentais

Table 1 - Sodium leves (\%) used in the experimental diets

\begin{tabular}{cc}
\hline $\begin{array}{c}\text { Período de repouso } \\
\text { Pause period }\end{array}$ & $\begin{array}{c}\text { Período de postura } \\
\text { Production period }\end{array}$ \\
\hline \multirow{2}{*}{0,15} & 0,15 \\
& 0,25 \\
0,25 & 0,35 \\
\hline & 0,15 \\
0,35 & 0,25 \\
& 0,35 \\
\hline \multirow{2}{*}{0,15} \\
& 0,25 \\
\end{tabular}


Tabela 2 - Composição das rações experimentais (\%)

Table 2 - Composition of the experimental diets (\%)

\begin{tabular}{|c|c|c|c|}
\hline \multirow[t]{2}{*}{$\begin{array}{l}\text { Ingredientes (\%) } \\
\text { Ingredients }\end{array}$} & \multicolumn{3}{|c|}{$\begin{array}{l}\text { Níveis de sódio (\%) } \\
\text { Sodium levels }\end{array}$} \\
\hline & 0,15 & 0,25 & 0,35 \\
\hline $\begin{array}{l}\text { Milho } \\
\text { Corn }\end{array}$ & 66,19 & 66,19 & 66,05 \\
\hline $\begin{array}{l}\text { Farelo de soja } \\
\text { Soybean meal }\end{array}$ & 22,88 & 22,88 & 22,90 \\
\hline $\begin{array}{l}\text { Calcário calcítico } \\
\text { Limestone }\end{array}$ & 7,99 & 7,99 & 7,99 \\
\hline $\begin{array}{l}\text { Fosfato bicálcico } \\
\text { Dicalcium phosphate }\end{array}$ & 1,54 & 1,54 & 1,54 \\
\hline $\begin{array}{l}\text { Areia lavada } \\
\text { Washed sand }\end{array}$ & 0,47 & 0,22 & 0,09 \\
\hline $\begin{array}{l}\text { Suplemento mineral+vitamínico } \\
\text { Mineral+ vitamin supplement }\end{array}$ & 0,50 & 0,50 & 0,50 \\
\hline $\begin{array}{l}\text { Sal } \\
\text { Salt }\end{array}$ & 0,29 & 0,54 & 0,79 \\
\hline $\begin{array}{l}\text { DL-metionina } 99 \\
\text { DL-methionine } 99\end{array}$ & 0,14 & 0,14 & 0,14 \\
\hline TOTAL & 100,00 & 100,00 & 100,00 \\
\hline $\begin{array}{l}\text { Composição calculada } \\
\text { Calculated composition }\end{array}$ & & & \\
\hline $\begin{array}{l}\text { Energia metabolizável (kcal/kg) } \\
\text { Metabolizable energy }\end{array}$ & 2800 & 2800 & 2800 \\
\hline $\begin{array}{l}\text { Proteína bruta }(\%) \\
\text { Crude protein }\end{array}$ & 16,07 & 16,07 & 16,06 \\
\hline $\begin{array}{l}\text { Fósforo total (\%) } \\
\text { Total phosphorus }\end{array}$ & 0,61 & 0,61 & 0,61 \\
\hline $\begin{array}{l}\text { Fósforo disponível (\%) } \\
\text { Available phosphorus }\end{array}$ & 0,39 & 0,39 & 0,39 \\
\hline $\begin{array}{l}\text { Sódio }(\%) \\
\text { Sodium }\end{array}$ & 0,15 & 0,25 & 0,35 \\
\hline $\begin{array}{l}\text { Cálcio (\%) } \\
\text { Calcium }\end{array}$ & 3,50 & 3,50 & 3,50 \\
\hline $\begin{array}{l}\text { Cloro }(\%) \\
\text { Chlorine }\end{array}$ & 0,20 & 0,36 & 0,51 \\
\hline $\begin{array}{l}\text { Potássio }(\%) \\
\text { Potassium }\end{array}$ & 0,44 & 0,44 & 0,44 \\
\hline $\begin{array}{l}\text { Metionina+cistina }(\%) \\
\text { Methionine + cystine }\end{array}$ & 0,68 & 0,68 & 0,68 \\
\hline $\begin{array}{l}\text { Metionina }(\%) \\
\text { Methionine }\end{array}$ & 0,40 & 0,40 & 0,40 \\
\hline $\begin{array}{l}\text { Lisina }(\%) \\
\text { Lysine }\end{array}$ & 0,79 & 0,79 & 0,79 \\
\hline $\begin{array}{l}\text { Treonina }(\%) \\
\text { Threonine }\end{array}$ & 0,63 & 0,63 & 0,63 \\
\hline $\begin{array}{l}\text { Triptofano }(\%) \\
\text { Tryptophan }\end{array}$ & 0,21 & 0,21 & 0,21 \\
\hline
\end{tabular}

${ }^{1}$ Suplemento mineral e vitamínico (Mineral and vitamin supplement). Quantidade/kg da dieta (Amount $/ \mathrm{kg}$ of diet): Vit.A - 1.500 UI; Vit. $D_{3}$ - 220 UI; Vit. E - 10 mg; Vit. K - 0,5 mg; Vit. B 2 mg; Vit. B 12 - 10 mcg; Pantotenato de cálcio (Calcium panthotenate) - 2,2 mg; Niacina (Niacin) - $10 \mathrm{mg}$; Bacitracina de zinco (Zinc bacitracin) - $50 \mathrm{mg}$; Colina (Choline) - $250 \mathrm{mg}$; Cobre (Copper) - $75 \mathrm{mg}$; lodo (lodine) - 0,3 mg; Selênio (Seleniun) - 0,1 mg; Manganês (Manganese) - 30 mg; Zinco (Zinc) - 50 mg; Ferro (Iron) - 50 mg; Butil Hidroxi Tolueno (Butil Hidroxi Toluen) $0,625 \mathrm{mg}$. 
Tabela 3 - Efeitos dos níveis de sódio sobre o desempenho e a qualidade do albúmen dos ovos de poedeiras comerciais no segundo ciclo de postura

Table 3 - Effect of sodium levels on performance and albumen quality of commercial laying hen eggs in the second phase of production

\begin{tabular}{|c|c|c|c|c|c|c|c|}
\hline $\begin{array}{l}\text { Sódio }(\%) \\
\text { Sodium }(\%)\end{array}$ & $\begin{array}{c}\text { Produção } \\
\text { ovos (\%) } \\
\text { Egg } \\
\text { production (\%) }\end{array}$ & $\begin{array}{c}\text { Peso } \\
\text { ovo }(\mathrm{g}) \\
\text { Egg } \\
\text { weight }(g)\end{array}$ & $\begin{array}{c}\text { Consumo } \\
\text { (g/ave/dia) } \\
\text { Intake } \\
\text { (g/bird/day) }\end{array}$ & $\begin{array}{c}\text { CA } \\
(\mathrm{kg} / \mathrm{kg}) \\
\text { Feed } \\
\text { conversion }\end{array}$ & $\begin{array}{c}\text { CA } \\
\text { (kg/dz) } \\
\text { Feed } \\
\text { conversion }\end{array}$ & $\begin{array}{c}\text { Massa ovo }(\mathrm{g}) \\
\text { Egg mass } \\
(\mathrm{g})\end{array}$ & $\begin{array}{l}\text { Unid. Haugh } \\
\text { Haugh unit }\end{array}$ \\
\hline \multicolumn{8}{|c|}{$\begin{array}{l}\text { Efeito dos níveis de sódio no período de repouso } \\
\text { Effect of sodium levels used in the pause period }\end{array}$} \\
\hline 0,15 & 83,36 & 64,96 & 109,21 & 2,02 & 1,57 & 54,16 & 90,43 \\
\hline 0,25 & 81,98 & 64,34 & 107,13 & 2,04 & 1,57 & 52,76 & 90,59 \\
\hline 0,35 & 79,17 & 65,62 & 107,16 & 2,07 & 1,63 & 51,95 & 90,45 \\
\hline \multicolumn{8}{|c|}{$\begin{array}{l}\text { Efeito dos níveis de sódio no período de postura } \\
\text { Effect of sodium levels used in the production period }\end{array}$} \\
\hline 0,15 & 81,66 & 64,83 & 109,37 & 2,07 & 1,61 & 52,94 & 91,14 \\
\hline 0,25 & 81,95 & 65,06 & 107,10 & 2,01 & 1,57 & 53,32 & 89,98 \\
\hline 0,35 & 80,90 & 65,04 & 107,03 & 2,04 & 1,60 & 52,62 & 90,34 \\
\hline \multicolumn{8}{|c|}{$\begin{array}{c}\text { Valores de } \mathrm{F} \\
\text { F values }\end{array}$} \\
\hline $\mathrm{A}^{2}$ & $0,90 \mathrm{~ns}$ & $1,40 \mathrm{~ns}$ & $0,26 \mathrm{~ns}$ & $0,39 \mathrm{~ns}$ & $0,87 \mathrm{~ns}$ & $0,72 \mathrm{~ns}$ & $0,068 \mathrm{~ns}$ \\
\hline $\mathrm{B}^{3}$ & $0,58 \mathrm{~ns}$ & $0,55 \mathrm{~ns}$ & $0,32 \mathrm{~ns}$ & $0,65 \mathrm{~ns}$ & $0,32 \mathrm{~ns}$ & $0,071 \mathrm{~ns}$ & $0,31 \mathrm{~ns}$ \\
\hline A x B & $0,36 \mathrm{~ns}$ & $1,46 \mathrm{~ns}$ & $0,63 \mathrm{~ns}$ & $0,66 \mathrm{~ns}$ & $0,35 \mathrm{~ns}$ & $0,86 \mathrm{~ns}$ & $0,96 \mathrm{~ns}$ \\
\hline $\mathrm{CV}^{3}(\%)$ & 6,78 & 2,03 & 5,33 & 4,21 & 5,22 & 6,12 & 2,89 \\
\hline
\end{tabular}

Médias na coluna, seguidas de letras iguais, não diferem $(P>0,05)$ pelo teste Tukey.

Means, within a column, followed by the same letters, are not significanty different (P>.05), according to Tukey test.

${ }^{*} \mathrm{P}<0,05 ;{ }^{* \star} \mathrm{P}<0,01$.

${ }^{1}$ Níveis de sódio fornecidos no período de repouso (Sodium levels used in the pause period).

${ }^{2}$ Níveis de sódio fornecidos no período de postura (Sodium levels used in the production period).

${ }^{3}$ Coeficiente de variação (\%) (Coefficient of variation).

Tabela 4 - Efeitos dos níveis de sódio sobre a qualidade da casca de ovo de poedeiras comerciais no segundo ciclo de postura

Table 4 - Effect of sodium levels on shell quality of commercial laying hen eggs, in the second phase of production

\begin{tabular}{cccc}
\hline $\begin{array}{c}\text { Sódio }(\%) \\
\text { Sodium }\end{array}$ & $\begin{array}{c}\text { \% Casca } \\
\text { \% Shell }\end{array}$ & $\begin{array}{c}\text { Espessura casca }(\mathrm{mm}) \\
\text { Shell thickness }-\mathrm{mm}\end{array}$ & $\begin{array}{c}\text { Gravidade específica } \\
\text { Specific gravity }\end{array}$ \\
\hline \multicolumn{4}{c}{ Efeito dos níveis de sódio no período de repouso } \\
Effect of sodium levels
\end{tabular}

Médias na coluna, seguidas de letras iguais, não diferem $(P>0,05)$ pelo teste Tukey.

Means, within a column, followed by the same letters, are not significanty different $(P>.05)$, according to Tukey test.

${ }^{*} \mathrm{P}<0,05$; ** $\mathrm{P}<0,01$.

1 Níveis de sódio fornecidos no período de repouso (Sodium levels used in the pause period).

2 Níveis de sódio fornecidos no período de postura (Sodium levels used in the production period).

${ }^{3}$ Coeficiente de variação (\%) (Coefficient of variation).

R. Bras. Zootec., v.33, n.2, p.391-396, 2004 
da casca e nenhuma outra característica relacionada à qualidade da casca. A gravidade específica não sofreu alteração $(\mathrm{P}>0,05)$ diante dos diferentes níveis de sódio, sendo que esse mesmo efeito também foi observado por Faria et al. (2000) e Junqueira et al. (2000). Figueiredo et al. (2001), trabalhando com cinco níveis de sódio $(0,13 ; 0,16 ; 0,19 ; 0,22$ e $0,25 \%)$ para poedeiras comerciais no segundo ciclo de postura, não encontraram efeito significativo para consumo de ração, produção e peso dos ovos, conversão alimentar, gravidade específica, percentagem e espessura da casca, concluindo que o nível de $0,13 \%$ de sódio na ração foi suficiente para atender à exigência nutricional de sódio de poedeiras no período pós-muda.

\section{Conclusões}

Os resultados deste experimento mostraram que o nível de $0,15 \%$ de sódio na ração foi suficiente para atender à exigência nutricional de sódio para poedeiras comerciais no segundo ciclo de postura, para melhores desempenho e qualidade dos ovos, entretanto, para garantir melhor espessura da casca, o nível de $0,25 \%$ seria o mais adequado.

\section{Literatura Citada}

ANDRIGUETTO, J.M.; PERLY, I.M.; GEMAEL, A. et al. Nutrição animal. 4.ed. São Paulo: 1986. v.1, 395p.

BURNS, C.H.; GRAVENS, W.W.; PHILLIPS, P.H. The requirement of breeding hens for sodium chloride. Poultry Science, v.31, p.302-306, 1952.

CHRISTMAS, R.B.; HARMS, R.H. The performance of four strains of laying hens subjected to various posttest combinations of calcium and phosphorus after forced rest in winter or summer. Poultry Science, v.62, n.9, p.1816-1822, 1983.

ESTAT 2.0 - Sistema de Análise Estatística. Jaboticabal: Pólo Computacional - Departamento de Ciências Exatas - UNESP. s.d.

FARIA, D.E.; JUNQUEIRA, O.M.; SAKOMURA, N.K. et al. Efeito de diferentes níveis de sódio e fósforo sobre o desempenho e a qualidade da casca dos ovos de poedeiras comerciais. Revista Brasileira Zootecnia, v.29, n.2, p.458-466, 2000.

FIGUEIREDO, D.F.; TORAL, F.L.B.; GARCIA, J. et al. Exigência nutricional de sódio para poedeiras pós-muda forçada. In: REUNIÃO ANUAL DA SOCIEDADE BRASILEIRA DEZOOTECNIA, 38., 2001, Piracicaba. Anais... Piracicaba: Sociedade Brasileira de Zootecnia, 2001. p.841.

HARMS, R.H. Effect of removing salt, sodium, or chloride from the diet of commercial layers. Poultry Science, v.70, n.2, p.333-336, 1991.

HOOGE, D.M. A importância dos eletrólitos. Avicultura Industrial, n.1068, p.20-26, 1999.
JUNQUEIRA, O.M.; CAMARGO-FILHO, B.; ARAUJO, L.F. et al. Efeitos das fontes e níveis de sódio, cloro e potássio e da relação $(\mathrm{Na}+\mathrm{K}) / \mathrm{Cl}$, sobre o desempenho e características do plasma sangüíneo de poedeiras comerciais. Revista Brasileira de Zootecnia, v.29, n.4, p.1110-1116, 2000.

KESHAVARZ, K.; NAKAJIMA, S. The effect of dietary manipulations of energy, protein and fat during the growing and laying periods on early egg weight and egg components. Poultry Science, v.74, n.11, p.1520-1528, 1995.

KESHAVARZ, K. Further investigations on the effect of dietary manipulations of nutrients on early egg weight. Poultry Science, v.74, n.1, p.50-61,1995.

KUCHINSKI, K.K.; HARMS, R.H.; RUSSEL, G. Re-evaluation of the sodium of the commercial laying hen. In: POULTRY SCIENCE ASSOCIATION ANNUAL MEETING, 86., 1997. Athens. Proceedings... Athens: Poultry Science, 1997. v.59, p.236. (Supplement 1)

LEESON, S.; SUMMERS, J.D. Commercial poultry nutrition. Guelph: University Books, 1991. 238p.

Mc DOWELL, L.R. Mineral in animal and human nutrition. San Diego: Academic Press, 1992. 524p.

MONGIN, P. Role of sodium, potassium and chloride in eggshell quality. In: FLORIDA NUTRITION CONFERENCE, 1980, Florida. Proceedings... Florida: 1980. p.213-223.

MORENG, R.E.; AVENS, J.S. Ciência e Produção de aves. São Paulo: Roca, 1990. 380p.

NATIONAL RESEARCH COUNCIL - NRC. Nutrient requirements of poultry. 8.ed. Washington, D.C.: National Academy of Science, $1984.71 \mathrm{p}$.

NATIONAL RESEARCH COUNCIL - NRC. Nutrient requirements of poultry. 8.ed. Washington, D.C.: National Academy of Science, 1994. 160p.

ROSTAGNO, H.S.; SILVA, D.J.; COSTA, P.M.A. et al. Composição de alimentos e exigências nutricionais (Tabelas brasileiras para aves e suínos). Viçosa, MG: Universidade Federal de Viçosa, 1985. 59p.

ROSTAGNO, H.S.; SILVA, D.J.; COSTA, P.M.A. et al. Composição de alimentos e exigências nutricionais (Tabelas brasileiras para aves e suínos). Viçosa, MG: Universidade Federal de Viçosa, 2000. 141p.

SCOTT, M.L.; NESHEIN, M.C.; YOUNG, R.J. Nutrition of the chicken. 3.ed. New York: Scott and Assoc. Publ., 1982. $562 \mathrm{p}$.

SCRAGG, R.H.; LOGAN, N.B.; GEDDES, N. Response egg weight to the inclusion of various fats in layer diets. British Poultry Science, v.28, n.1, p.15-21. 1987.

WIDEMAN, R.F.; BUSS, E.G. Arterial blood gas, $\mathrm{pH}$ and bicarbonate values in laying hens selected for thick or thin eggshell production. Poultry Science, v.64, n.5, p.1015-1019, 1985.

ZIMMERMAN, R.A. Management of egg size through precise nutrient delivery. Journal of Applied Poultry Research, v.6, n.4, p.476-482, 1997. 